\title{
Living with primary immunodeficiency disease during the Covid-19 pandemic
}

\author{
Kerri L. Sowers ${ }^{1}$ (D) $\cdot$ Mary Lou Galantino ${ }^{1,2,3}$ \\ Received: 28 September 2020 / Accepted: 6 April 2021 / Published online: 26 April 2021 \\ (C) The Author(s), under exclusive licence to Springer-Verlag GmbH Germany, part of Springer Nature 2021
}

\begin{abstract}
Aim The purpose of this survey study was to investigate the impact of Covid-19 on the lives of individuals living with primary immunodeficiency disease (PID).

Subject and methods An online survey was distributed through social media to individuals with a diagnosis of PID to investigate behaviors and concerns during the Covid-19 pandemic.

Results Five hundred and fifty seven responses were collected, of which 495 surveys were $100 \%$ complete; partial responses were analyzed. Respondents have been extremely cautious and have minimized their potential Covid-19 exposure risk. In this study, 56.6\% $(n=289)$ participated in telehealth visits with the physician responsible for managing their PID during the Covid-19 pandemic. Respondents reported they would be somewhat comfortable with returning to normal activities if there was widespread herd immunity $(40.9 \%, n=209)$, an effective vaccine $(46.0 \% . n=235)$, or public health protections $(44.0 \%, n=225)$. The majority of respondents were extremely concerned $(57.3 \%, n=293)$ about additional waves of Covid-19 cases when their state or country reopens.

Conclusion The PID community is aware of the health risks posed by this public health crisis, and have done as much as possible to minimize their risk to community exposure. This pandemic has highlighted the importance of continuous medical care for a vulnerable population through the use of telemedicine. Healthcare providers should be aware of the emotional burden and increased psychiatric distress, often presenting as fear, anxiety, or depression, in patients with a chronic medical condition during a public health crisis such as the Covid-19 pandemic.
\end{abstract}

Keywords Primary immunodeficiency disease $\cdot$ Common variable immune deficiency $\cdot$ Covid-19 $\cdot$ SARS-CoV-2 $\cdot$ Pandemic $\cdot$ Chronic health condition · Anxiety $\cdot$ Public health

\section{Background}

In December of 2019, a cluster of pneumonia cases in Wuhan, China initiated the severe acute respiratory syndrome coronavirus 2, or SARS-CoV-2, pandemic (Fu et al. 2020). On January 30, 2020, the World Health Organization declared a

Kerri L. Sowers

Kerri.Sowers@stockton.edu

Mary Lou Galantino

MaryLou.Galantino@stockton.edu

1 Stockton University, 101 Vera King Farris Drive, Galloway, NJ 08205, USA

2 University of Pennsylvania, Philadelphia, PA 19104, USA

3 University of Witwatersrand, Johannesburg, South Africa
Public Health Emergency of International Concern (Hu et al. 2020). This novel coronavirus, also referred to as Covid-19, causes symptoms such as fever, cough, fatigue, and dyspnea (Fu et al. 2020; Hu et al. 2020). Typical laboratory findings with Covid-19 include elevated C-reactive protein, decreased lymphocyte count, and increased lactate dehydrogenase (Fu et al. 2020). Patients who develop significant lymphopenia, elevated $\mathrm{C}$ reactive protein, elevated procalcitonin, elevated serum ferritin, and elevated interleukin-6 (IL-6) have a much poorer prognosis (Terpos et al. 2020; Tian et al. 2020; Zheng et al. 2020). The presence of comorbidities, such as hypertension, coronary heart disease, and diabetes, are associated with a significantly high risk of death in individuals diagnosed with Covid-19 (Tian et al. 2020; Zheng et al. 2020). The estimated reproduction number for the virus is between 1.9 to 6.5 , with most studies estimating an $\mathrm{R}_{0}$ of 2.0-3.0 (Park et al. 2020). Case fatality rates remain unknown, with modeling estimates 
from $0.3 \%$ to $1.4 \%$ (Park et al. 2020). The mortality rates of SARS-CoV-2 are lower than that of SARS or Middle East Respiratory Syndrome (MERS), but higher than the seasonal flu (Terpos et al. 2020).

In addition to the physiological impact of Covid-19, there is also a psychological impact felt by the global community. The risk of disease, concern about friends or family, job and food insecurity, lifestyle alterations, quarantine, and social isolation all pose threats to mental well-being and add to the stress during this global pandemic. Covid-19 is expanding the number of risk factors for the development of psychiatric symptoms, including quarantine and isolation, concerns about family and friends contracting the virus, and altered living or work conditions (Vindegaard and Benros 2020). Health crises, such as the Covid-19 pandemic, can lead to psychological changes caused by fear, anxiety, depression, or insecurity (Salari et al. 2020).

A systematic review and meta-analysis by Luo et al. (2020) noted that the Covid-19 pandemic has had a high psychological impact on healthcare providers, the general public, and individuals with pre-existing conditions, or those diagnosed with Covid-19. Anxiety and depression were the most commonly reported psychological impacts; individuals with preexisting conditions or who were diagnosed with Covid-19 reported significantly higher levels of anxiety and depression (Vindegaard and Benros 2020; Luo et al. 2020). Posttraumatic stress syndrome (PTSS) was found to be very high, $96.2 \%$, for patients admitted to the hospital with Covid-19, as compared to $7 \%$ in the general population (Vindegaard and Benros 2020). Salari et al. (2020) noted an association between a higher education level and increased anxiety, depression, and stress during the Covid-19 pandemic. Furthermore, individuals with chronic disease are already at a higher risk for increased psychiatric distress, and the Covid-19 pandemic may cause a sense of greater vulnerability (Salari et al. 2020).

Infections, autoimmune conditions, inflammatory disorders, and malignancies are hallmarks of rare genetic conditions known as primary immunodeficiencies (PIDs) (Tangye et al. 2020). The 2019 update from the International Union of Immunological Societies Expert Committee (IUIS) identified 430 inborn errors of immunity that fall into the category of a PID (Tangye et al. 2020). There is limited information about the prevalence of PID either in the United States or globally. A phone survey of 10,000 households conducted by Boyle and Buckley (2007) estimated the PID prevalence to be 8.33 per 10,000 or $1: 1200$ (approximately $150,000-360,000$ cases in the United States). Kobrynski et al. (2014) completed a retrospective review of healthcare databases in the United States and found the prevalence of PID to increase from 38.9 to 50.5 per 100,000 (private insurance) and from 29.1 to 41.1 per 100,000 (public insurance) during the period of 2001 to 2007. In this review, the older age groups $(45-54,55-$ 64 years) had a higher prevalence for all PID diagnoses compared with the population aged 21 and younger, and B cell defects were most reported (Kobrynski et al. 2014). Kobrynski et al. (2014) also noted that prevalence was more than twice as high among Whites as compared to Blacks or Hispanics. There is great variability in the reported gender prevalence among various publications and medical databases. Leung et al. (2018) notes that adult-onset common variable immune deficiency (CVID) is more common in women, while instances of pediatric-onset PID are more often higher in males due to $\mathrm{x}$-linked disorders. Most disease registries or databases show a higher number of males (Leung et al. 2018), as was the case with the survey conducted by Boyle and Buckley (2007). However, the retrospective review by Kobrynski et al. (2014) showed a higher prevalence of females for overall PID cases.

There is little research to date about the impact of Covid-19 on the PID community. Pulvirenti et al. (2020) conducted a study on the health-related quality of life (HRQoL) in patients with CVID who were switched to home-based therapies and telemedicine visits due to the Covid-19 pandemic. The percentage of patients who were found to be at risk for anxiety or depression was similar for those who were forced to switch to home-based subcutaneous immunoglobulin (SCIG) therapy as compared to those who were already using a home therapy (Pulvirenti et al. 2020). Pulvirenti et al. (2020) noted that the Covid-19 pandemic did have a negative impact on the HRQoL in their patients, but there was no negative impact on HRQoL from switching to home-based therapy and telemedicine.

Chronic medical conditions create lifelong challenges, and more so during times of ambiguity. Patients must learn to cope with the physiological issues, emotional adjustments, lifestyle adaptations, and adverse consequences (de Ridder et al. 2008). PIDs are chronic, and patients must learn to cope with their diagnosis and the ongoing management of their medical condition. Add the challenges associated with a global pandemic, due to Covid-19, and the burden on the PID community is exponentially heightened. Therefore, the purpose of this survey study was to investigate the impact of Covid-19 on the lives of individuals living with a diagnosis of PID.

\section{Methodology}

This research was approved by the Institutional Review Board at Stockton University. Informed consent was obtained through the first question of the online survey. Individuals with a diagnosis of PID who were 18 years or older were eligible to participate. The survey was distributed to individuals with a diagnosis of PID through multiple public and private social media patient support groups that primarily target patients in the United States. 
The Qualtrics survey platform was used to create and distribute the survey. Data analysis was done using SPSS version 25 . The survey was available through an anonymous link during May and June of 2020. Respondents were asked to complete the survey only one time. It was estimated that it would take respondents 15 to $20 \mathrm{~min}$ to complete the survey. Some questions allowed only one response while others permitted multiple responses. Questions in the survey were presented in the same order for each respondent. The survey was designed to be compatible with any mobile device (such as a tablet or smartphone); survey respondents used seven pages to reach the end of the survey. Respondents were able to change prior responses, and could return to the survey if they were unable to complete it in one sitting. The survey consisted of a consent acknowledgment question and 21 survey questions, two with multiple parts. To improve face validity, the survey was initially sent to several individuals with a diagnosis of PID, to test the survey platform and provide feedback on survey flow. The survey consisted of initial demographic questions followed by questions to assess behaviors and concerns during the Covid-19 pandemic.

\section{Results}

A total of 557 responses were collected; of those, 495 surveys were $100 \%$ complete. Analysis of 511 surveys will be discussed; partially completed surveys were included in the data analysis, provided they answered at least one question beyond the demographic assessment. There were 46 surveys not included in the data analysis as they either opted to answer $n o$ for the informed consent question or they only completed the demographic questions. Respondent demographics are presented in Table 1.

\section{Exposure}

Respondents were asked to describe their exposure to Covid19. Of the 511 responses, $1.4 \%(n=7)$ reported they were/had been diagnosed positive for Covid-19; 8.8\% $(n=45)$ reported that they currently had or had had symptoms consistent with Covid-19, but had not been able to access testing, were awaiting test results, or had a negative result; $13.3 \%(n=68)$ reported no symptoms for Covid-19, but may have been exposed to the virus; and $76.5 \%(n=391)$ reported no symptoms and they did not think they had been exposed to the virus.

\section{Worry}

Respondents were asked how worried they were that they, or a close family member, would contract Covid-19. Of the 511
Table 1 Demographics

\begin{tabular}{lc}
\hline Respondents & $N=511$ \\
\hline Gender \%(n) & \\
Male & $5.5(28)$ \\
Female & $93.9(480)$ \\
Non-binary & $0.2(1)$ \\
Prefer not to identify & $0.4(2)$ \\
Age \%(n) & \\
18-29 years & $9.8(50)$ \\
30-44 years & $31.3(160)$ \\
45-54 years & $21.9(112)$ \\
55-64 years & $23.1(118)$ \\
65-74 years & $12.3(63)$ \\
75 years and older & $1.6(8)$ \\
Race/ethnicity \%(n) & \\
White/Caucasian & $91.2(466)$ \\
Hispanic/Latino & $1.6(8)$ \\
Black/African American & $0.6(3)$ \\
Asian & $0.4(2)$ \\
Native American/Alaskan Native & $0.4(2)$ \\
Other/multiple ethnicities & $5.9(30)$ \\
Type of PID \%( $n$ ) & \\
CVID & $79.8(408)$ \\
SAD & $4.9(25)$ \\
SCID & $0.8(4)$ \\
IgG subclass deficiency & $7.4(38)$ \\
Agammaglobulinemia & $1.0(5)$ \\
Selective IgA deficiency & $1.0(5)$ \\
Hyper IgM deficiency & $0.6(3)$ \\
CGD & $0.2(1)$ \\
Complement deficiency & $0.4(2)$ \\
Other/unsure & $3.9(20)$ \\
\hline
\end{tabular}

$\mathrm{CVID}=$ common variable immune deficiency $\mathrm{SAD}=$ specific antibody deficiency; SCID = severe combined immune deficiency; $\mathrm{CGD}=$ chronic granulomatous disease

responses, $30.9 \%(n=158)$ were extremely concerned; $33.3 \%$ $(n=170)$ were very concerned; $26.6 \%(n=136)$ were moderately concerned; $7.6 \%(n=39)$ were slightly concerned; and $1.6 \%(n=8)$ were not concerned at all.

\section{Ability to function}

Respondents were asked if anxiety or concern about Covid-19 has impacted their daily life or ability to function. Of the 511 responses, $16.8 \%(n=86)$ indicated constantly; $22.3 \%(n=$ $114)$ indicated very frequently; $24.5 \%(n=125)$ indicated frequently; $23.3 \%(n=119)$ indicated occasionally; $10.0 \%$ $(n=51)$ indicated rarely; $3.1 \%(n=16)$ indicated not at all. 


\section{Change in attitude or behaviors}

Respondents were asked about their level of anxiety or concern about being exposed to viruses/illness when in the community (for activities such as shopping or going to medical appointments) before and after the start of the Covid-19 pandemic; the response options were from extremely high to extremely low, using a 5-point Likert scale. Of the 511 responses, 3.9\% $(n=20)$ indicated extremely high; $27.0 \%(n=$ $138)$ indicated high; $36.2 \%(n=185)$ indicated neither high nor low; $22.9 \%(n=117)$ indicated low; and $10.0 \%(n=51)$ indicated extremely low regarding concern about exposure to illness in the community, prior to the pandemic. In comparison, the 511 responses were much different when asked about their concern for exposure to illness in the community during the pandemic, where $46.2 \%(n=236)$ indicated extremely high; 38.6\% $(n=197)$ indicated high; 10.4\% $(n=53)$ indicated neither high nor low; $3.3 \%(n=17)$ indicated low; and $1.6 \%(n=8)$ indicated extremely low.

Respondents were also asked about their level of caution when out in their community (for work, shopping, or medical appointments). They could select from: no concerns (did not take any special precautions); minimal concerns (used hand sanitizer, avoided large crowds, more frequent hand washing); moderate concerns (used a mask, avoided most crowds, wiped down items before bringing them into the home); or significant concerns (avoided leaving the house unless absolutely necessary). Of the 511 responses, $21.1 \%(n=108)$ indicated no concern; $63.0 \%(n=322)$ indicated minimal concern; $12.5 \%(n=64)$ indicated moderate concern; and $3.3 \%(n=$ 17) indicated significant concern regarding their level of caution when out in their community, prior to the pandemic. In comparison, the 511 responses were much different when asked about their caution when out in the community during the pandemic, where $0.8 \%(n=4)$ indicated no concerns; 4.7\% $(n=24)$ indicated minimal concerns; $37.0 \%(n=189)$ indicated moderate concerns; and 57.5\% ( $n=294)$ indicated significant concerns.

A Wilcoxon signed-rank test, using an alpha of 0.05 , showed that there was a statistically significant change in anxiety about being exposed to viruses or infections when in the community (going shopping or to medical appointments) in individuals from before the Covid-19 pandemic as compared to during the pandemic $(Z=-17.206$, $p<0.001$ ). Median anxiety rating was 3 (representing neither low nor high) for before the pandemic and 2 (representing high) for during the pandemic. A Wilcoxon signed-rank test, using an alpha of 0.05 , showed that there was a statistically significant change in level of caution when out in the community (working, going shopping, or going to medical appointments) in individuals from before the Covid-19 pandemic as compared to during the pandemic $(Z=-18.894, p<0.001)$.
Median concern rating was 2 (representing minimal concern) for before the pandemic and 4 (representing significant concern) during the pandemic.

\section{Physical activity}

Respondents were asked to compare their current level of physical activity to before the start of the pandemic. Of the 505 respondents, $8.6 \%(n=44)$ indicated much more; $14.3 \%$ $(n=73)$ indicated slightly more; $26.8 \%(n=137)$ indicated the same; $21.7 \%(n=111)$ indicated slightly less; and $27.4 \%(n=$ 140 ) indicated much less regarding their level of physical activity as compared to before the start of the pandemic.

Respondents were also asked if they felt they were able to safely participate in physical activity without increasing their risk of exposure to Covid-19. Of the 505 respondents, $23.9 \%$ $(n=122)$ indicated very safe; $27.8 \%(n=142)$ indicated somewhat safe; $13.7 \%(n=70)$ indicated neither safe nor risky; $23.1 \%(n=118)$ indicated somewhat risky; and $10.4 \%(n=$ 53) indicated very risky regarding the safety of physical activity during the pandemic.

\section{Restrictions and social distancing}

Respondents were asked about their level of concern with the easing of restrictions and reduction of social distancing rules and guidelines. Of the 505 responses to this question, $43.2 \%$ $(n=221)$ were extremely concerned; $29.2 \%(n=149)$ were very concerned; $16.4 \%(n=84)$ were moderately concerned; $8.6 \%(n=44)$ were slightly concerned; and $1.4 \%(n=7)$ were not concerned at all.

\section{Social distancing practice}

Respondents were asked to describe the degree of social distancing or isolation they have practiced during the Covid-19 pandemic. Of the 495 responses to this question, $28.4 \%$ ( $n=$ 145 ) have been in complete isolation with no community exposure; $57.1 \%(n=292)$ have stayed mostly isolated, with only minimal exposure for shopping or medical appointments; $7.6 \%(n=39)$ have been isolated with moderate exposure for shopping, medical appointments, or other activities; $3.7 \%$ $(n=19)$ have had significant community exposure due to job requirements, a family member's community exposure, or exposure due to other reasons.

\section{Physician contact}

Respondents were asked whether they were contacted by the physician who is responsible for managing their PID about special precautions they should take during the Covid-19 pandemic. Of the 505 responses to this question (respondents were allowed to select multiple responses to this question), 
$8.0 \%(n=41)$ reported receiving a generic form letter or generic phone call from the physician's office; $14.9 \%(n=76)$ reported being personally contacted by their physician; $22.1 \%$ $(n=113)$ reported a face-to-face meeting with their physician via telehealth; and $56.0 \%(n=286)$ reported that they were not contacted by the physician responsible for managing their PID (or by the physician's office).

\section{Routine medical appointments}

Respondents were asked if they have been able to attend routine medical appointments during the Covid-19 pandemic (multiple responses were permitted for this question. Of the 505 responses to this question, $56.6 \%(n=289)$ have participated in telehealth visits; $32.1 \%(n=164)$ have visited a provider's office; $8.4 \%(n=43)$ have been unable to access their provider due to the office being closed, having restricted access, or not offering telehealth services; and $21.5 \%(n=110)$ have opted to not visit their medical provider due to concerns about the risk of potential Covid-19 exposure.

\section{Non-routine medical care}

Respondents were asked whether they have needed to seek care during the Covid-19 pandemic for non-routine medical care (multiple responses were permitted for this question). Of the 504 responses to this question, 37.6\% $(n=192)$ obtained non-routine medical care via telehealth resources; $21.5 \%(n=$ 110) obtained non-routine medical care through a physical visit to a provider office, emergency room, or urgent care; $4.3 \%(n=22)$ were not able to obtain non-routine care due to access issues during the pandemic; $18.0 \%(n=92)$ reported avoiding seeking out non-routine care due to concerns about the risk of Covid-19 exposure; and 30.5\% $(n=156)$ reported they did not need to seek out non-routine medical care during this time.

\section{Confidence in healthcare}

Respondents were asked to share their level of comfort in going to routine medical appointments and for emergency care with the standard level of healthcare practices (those in place prior to the Covid-19 pandemic). Of the 500 responses, $5.5 \%(n=28)$ indicated extremely comfortable; $13.3 \%(n=$ $68)$ indicated somewhat comfortable; $5.5 \%(n=28)$ indicated neither comfortable nor uncomfortable; $33.3 \%(n=170)$ indicated somewhat uncomfortable; and $40.3 \%(n=206)$ indicated extremely uncomfortable regarding the use of standard precautions for routine medical care. The same 500 respondents were then asked about standard precautions for emergency medical care: $6.1 \%(n=31)$ indicated extremely comfortable; $7.8 \%(n=40)$ indicated somewhat comfortable; $5.5 \%(n=28)$ indicated neither comfortable nor uncomfortable; $26.4 \%(n=$
$135)$ indicated somewhat uncomfortable; and $52.1 \%(n=266)$ indicated extremely uncomfortable.

Respondents were asked to share their level of comfort in going to routine medical appointments and for emergency care with enhanced precautions (including mask use, social distancing, additional testing). Of the 500 responses, $8.0 \%$ $(n=41)$ indicated extremely comfortable; $36.2 \%(n=185)$ indicated somewhat comfortable; $7.8 \%(n=40)$ indicated neither comfortable nor uncomfortable; $33.3 \%(n=170)$ indicated somewhat uncomfortable; and $12.5 \%(n=64)$ indicated extremely uncomfortable regarding the use of enhanced precautions for routine medical care. The same 500 respondents were then asked about enhanced precautions for emergency medical care: $7.2 \%(n=37)$ indicated extremely comfortable; $28.4 \%(n=145)$ indicated somewhat comfortable; $5.7 \%(n=$ $29)$ indicated neither comfortable nor uncomfortable; $31.9 \%$ $(n=163)$ indicated somewhat uncomfortable; and $24.7 \%(n=$ 126) indicated extremely uncomfortable.

\section{Return to normal activities}

Respondents were asked how comfortable they would feel about returning to their normal activities and community interactions if there was widespread herd immunity to Covid-19, if there was an effective vaccine available for Covid-19, or if their country/state continued to enforce public health protections, including social distancing, mandatory mask use, and restrictions on crowds/gatherings. Regarding widespread herd immunity, 497 respondents indicated they were: extremely comfortable, $8.0 \%(n=41)$; somewhat comfortable, $40.9 \%$ $(n=209)$; neither comfortable nor uncomfortable $12.7 \%$ $(n=65)$; somewhat uncomfortable, $25.6 \%(n=131)$; and extremely uncomfortable, $10.0 \%(n=51)$. The same 497 respondents were then asked about returning to activities with an effective vaccine: $11.7 \%(n=60)$ indicated extremely comfortable; $46.0 \%(n=235)$ indicated somewhat comfortable; $14.5 \%(n=74)$ indicated neither comfortable nor uncomfortable; $20.2 \%(n=103)$ indicated somewhat uncomfortable; and $4.9 \%(n=25)$ indicated extremely uncomfortable. Finally, the 497 respondents were asked about returning to activities with widespread public health protections: $10.0 \%(n=51)$ indicated extremely comfortable; $44.0 \%$ $(n=225)$ indicated somewhat comfortable; $15.3 \%(n=78)$ indicated neither comfortable nor uncomfortable; $20.7 \%(n=$ $106)$ indicated somewhat uncomfortable; and $7.2 \%(n=37)$ indicated extremely uncomfortable.

\section{Additional outbreaks}

Respondents were asked to share how concerned they are about additional waves of Covid-19 outbreaks or about having an increased number of cases on Covid-19 when their state/ country reopens. Of the 495 responses to this question, $57.3 \%$ 
$(n=293)$ were extremely concerned; $22.7 \%(n=116)$ were very concerned; $11.7 \%(n=60)$ were moderately concerned; $4.1 \%(n=21)$ were slightly concerned; and $1.0 \%(n=5)$ were not concerned at all.

\section{Discussion}

The results of this survey provide valuable information about the impact of the Covid-19 pandemic on the lives and behaviors of individuals diagnosed with PID. The majority of respondents did not report symptoms of Covid-19 and believed they had not been exposed to Covid-19. This corresponds to the response that most individuals remained isolated, with no community exposure, or were mostly isolated, with only minimal exposure for shopping or medical appointments. The respondents in this survey have been extremely cautious and have minimized their potential Covid-19 exposure risk.

The respondents did report a high level of concern that they or a close family member would contract Covid- 19 . Furthermore, they reported a high level of anxiety about Covid-19 that has impacted their daily life or ability to function. Individuals with a chronic disease are at a higher risk for anxiety and depression, which may be exacerbated by the current pandemic (Salari et al. 2020). There was a statistically significant change $(p<0.001)$ in the respondents' anxiety levels about their risk of being exposed to viruses or infections when engaged in community activities prior to the pandemic as compared to during the pandemic. Despite a typical high susceptibility to infections, only $3.9 \%(n=20)$ reported extremely high concern about community exposure to pathogens before the pandemic. However, $46.2 \%(n=236)$ reported extremely high concern about exposure during the Covid-19 pandemic. There was also a statistically significant change $(p<0.001)$ in the respondents' level of caution when out in the community, with only $3.3 \%(n=17)$ having a significant concern before the pandemic, as compared to $57.5 \%(n=294)$ during the Covid-19 pandemic. These findings are consistent with anxiety found in other patient populations during the pandemic (Torales et al. 2020).

Interestingly, 56.0\% $(n=286)$ of respondents reported that they were not contacted by the physician responsible for managing their PID (or by the physician's office). Effective and timely communication of risk by physicians during a public health crisis is critically important (Ledford and Anderson 2020). By providing factual information and guiding patients in best practices, physicians can reduce the emotional burden on their patients due to, and during, the Covid-19 pandemic (Ledford and Anderson 2020).

The Covid-19 pandemic has created a new opportunity for the expansion of the implementation of telemedicine as a routine practice. In this study, 56.6\% $(n=289)$ participated in telehealth visits with the physician responsible for managing their PID during the Covid-19 pandemic, while $21.5 \%(n=$ 110) have opted to not visit their medical provider due to concerns about the risk of potential Covid-19 exposure. Furthermore, $37.6 \%(n=192)$ obtained non-routine medical care via telehealth resources, while $18.0 \%(n=92)$ reported avoiding seeking out non-routine care due to concerns about the risk of Covid-19 exposure. Telemedicine is a critically important option to allow for continuity of care in the event of a public health crisis. Virtual visits help accommodate overscheduled clinics, reduce travel burden on patients, increase adherence of monitoring chronic conditions, and allow for uninterrupted care for vulnerable and immunodeficient patients (Keswani et al. 2020). There was no clear trend in the responses for how comfortable the respondents would be with enhanced precautions (including mask use, social distancing, and additional testing) in order to return to inperson medical appointments or emergency care. However, the majority of respondents reported they would feel extremely uncomfortable if only standard precautions were in place.

The respondents in this study showed a trend towards less physical activity during the Covid-19 pandemic (Stanton et al. 2020). Physical activity helps to reduce overall cardiovascular risks, which are risk factors in the most severe cases of Covid19 (Dwyer et al. 2020). Prior to the Covid-19 pandemic, inadequate physical activity was already seen as a global public health concern (Martinez-Ferran et al. 2020). Martinez-Ferran et al. (2020) described how quarantine has further contributed to a lack of physical activity (closure of exercise facilities, less community walking, limited education to support a home exercise program) and nutritional changes (limited access to stores, income limitations impacting choice of food quality, and overeating due to stress). There was a diverse opinion from the respondents about the safety of exercise during the Covid-19 pandemic; this is likely due to the type and location of exercise activities (i.e., homebased exercise programs could be continued uninterrupted, while respondents who accessed a public gym may perceive a higher exposure risk or may not have had access due to closure of the facility). Home-based or socially distant physical activity should be encouraged during the Covid-19 pandemic, with exercise frequency, duration, and intensity tailored to the individual's level of fitness and physical ability (Dwyer et al. 2020). Furthermore, physical activity contributes to improving psychological well-being, by enhancing self-esteem and resilience to stress and reducing depression and anxiety (Maugeri et al. 2020). It is critically important to encourage individuals to engage in routine exercise during the Covid-19 pandemic, as it will contribute to improving both physical and mental health.

Respondents reported they would be somewhat comfortable with returning to normal activities if there was widespread herd immunity $(40.9 \%, n=209)$, an effective vaccine $(46.0 \% . n=235)$, or public health protections including social distancing, mandatory mask use, and restrictions on crowds/ gatherings $(44.0 \%, n=225)$. Ultimately, the majority of 
respondents were extremely concerned $(57.3 \%, n=293)$ about additional waves of Covid-19 cases when their state or country reopens. Researchers agree that achieving global herd immunity is unlikely without widespread vaccination (Chen et al. 2020; Kwok et al. 2020; Randolph and Barreiro 2020). Conservative epidemiological estimates using a uniform herd immunity threshold of $67 \%\left(\mathrm{R}_{0}=3\right)$ and an infection fatality rate of $0.6 \%$, the absolute number of expected deaths across the globe would exceed 30 million people (Randolph and Barreiro 2020). Clearly, a natural pathway to herd immunity is unacceptable. The respondents in this study have cause for concern about additional waves of Covid-19. The Covid-19 pandemic has been modeled as a series of subepidemics, presenting in multiple waves, influenced by public health intervention measures (Kaxiras and Neofotistos 2020). He et al. (He et al. 2020) found similar epidemiologic characteristics between the current Covid-19 pandemic and the 1918-1919 influenza pandemic, suggesting that additional waves of the virus will be impacting the global population. Many countries are relaxing restrictions because they have to weigh ethical issues and social and economic crises against another potential Covid-19 wave (Wang et al. 2020). Premature relaxation of strict interventions is likely to lead to additional waves of Covid-19 infections (Xu and Li 2020). Ending the Covid-19 pandemic will require continued efforts by individuals, communities, and public health officials (Wang et al. 2020).

One limitation of this survey is the high percentage of white females with a diagnosis of CVID. This is likely due to the sampling method as the survey was posted on multiple social media sites for PID support. Social media is very useful due to its speed, low cost, and accessibility to reach less common diagnoses, but there is a tendency for social media based sampling to have an overrepresentation of young, white females (Whitaker et al. 2017). This survey did have a wide range of age groups. Another limitation was that this survey was not a validated tool, though it was assessed for face validity by sending it to several individuals with a diagnosis of PID prior to distribution. The responses to the survey could also be influenced by the local impact of the Covid-19 pandemic. Throughout the United States during May and June, there was great variability in the impact of the virus; some states had a small number of cases with minimal community restrictions, while other states had high rates of infection and mortality with a strict lockdown. The local impact of the public health crisis may have an impact on the responses to individual questions on this survey.

\section{Conclusion}

This research study provides insight into the impact of the Covid-19 pandemic on the lives and behaviors of individuals with a diagnosis of PID. The responses suggest that members of the PID community are very aware of the health risks posed by this public health crisis and have done as much as possible to minimize their risk to community exposure. This pandemic has highlighted the importance of continuous medical care for a vulnerable population through the use of telemedicine. Healthcare providers must be aware of the emotional burden and increased psychiatric distress, often presenting as fear, anxiety, or depression, in patients with a chronic medical condition during a public health crisis such as the Covid-19 pandemic. Thus, best practices in telemedicine are necessary to optimize patient care during pandemic uncertainties (Loeb et al. 2020). Patients may require additional communication, support, and education during these challenging times.

Acknowledgements The authors would like to thank the individuals in the PID community for their enthusiastic participation in this study.

Declarations This research study was IRB approved by Stockton University.

Conflict of interest The authors declare they have no conflict of interest.

\section{References}

Boyle JM, Buckley RH (2007) Population prevalence of diagnosed primary immunodeficiency diseases in the United States. J Clin Immunol 27(2007):497-502. https://doi.org/10.1007/s10875-0079103-1

Chen J, Ye L, Zhou MY, Cheng YR, Wang MW, Feng ZH (2020) Herd immunity and COVID-19. Eur Rev Med Pharmacol Sci 24(8): 4064 4065. https://doi.org/10.26355/eurrev_202004_20978

de Ridder D, Geenen R, Kuijer R, van Middendorp H (2008) Psychological adjustment to chronic disease. Lancet 372(9634): 246-255. https://doi.org/10.1016/S0140-6736(08)61078-8

Dwyer MJ, Pasini M, De Dominicis S, Righi E (2020) Physical activity: benefits and challenges during the COVID-19 pandemic. Scand J Med Sci Sports 30(7):1291-1294. https://doi.org/10.1111/sms. 13710

Fu L, Wang B, Yuan T, Chen X, Ao Y, Fitzpatrick T (2020) Clinical characteristics of coronavirus disease 2019 (COVID-19) in China: a systematic review and meta-analysis. J Inf Secur 80(6):656-665. https://doi.org/10.1016/j.jinf.2020.03.041

He D, Zhao S, Li Y, Cao P, Gao D, Lou Y et al (2020) Comparing COVID-19 and the 1918-19 influenza pandemics in the United Kingdom. Int J Infect Dis 98:67-70. Advance online publication. https://doi.org/10.1016/j.ijid.2020.06.075

Hu Y, Sun J, Dai Z, Deng H, Li X, Huang Q et al (2020) Prevalence and severity of corona virus disease 2019 (COVID-19): a systematic review and meta-analysis. J Clin Virol 127:104371. https://doi.org/ 10.1016/j.jcv.2020.104371

Kaxiras E, Neofotistos G (2020) Multiple epidemic wave model of the COVID-19 pandemic: modeling study. J Med Internet Res 22(7): e20912. https://doi.org/10.2196/20912

Keswani A, Brooks JP, Khoury P (2020) The future of telehealth in allergy and immunology training. J Allergy Clin Immunol Pract 8(7):2135-2141. https://doi.org/10.1016/j.jaip.2020.05.009

Kobrynski L, Powell RW, Bowen S (2014) Prevalence and morbidity of primary immunodeficiency diseases, United States 2001-2007. J 
Clin Immunol 34(8):954-961. https://doi.org/10.1007/s10875-014$0102-8$

Kwok KO, Lai F, Wei WI, Wong S, Tang J (2020) Herd immunity estimating the level required to halt the COVID-19 epidemics in affected countries. J Inf Secur 80(6):e32-e33. https://doi.org/10. 1016/j.jinf.2020.03.027

Ledford CJW, Anderson LN (2020) Communication strategies for physicians practicing throughout emerging public health crises. Fam Med 52(5):48-50. https://doi.org/10.22454/FamMed.2020.960734

Leung J, Sullivan KE, Perelygina L, Icenogle JP, Fuleihan RL, Lanzieri TM (2018) Prevalence of granulomas in patients with primary immunodeficiency disorders, United States: data from national health care claims and the US Immunodeficiency Network registry. J Clin Immunol 38(6):717-726. https://doi.org/10.1007/s10875-0180534-7

Loeb AE, Rao SS, Ficke JR, Morris CD, Riley L, Levin AS (2020) Departmental experience and lessons learned with accelerated introduction of telemedicine during the COVID-19 crisis. J Am Acad Orthop Surg 28(11):e469-e476. https://doi.org/10.5435/JAAOS-D20-00380

Luo M, Guo L, Yu M, Jiang W, Wang H (2020) The psychological and mental impact of coronavirus disease 2019 (COVID-19) on medical staff and general public - a systematic review and meta-analysis. Psych Res 291:113190. Advance online publication. https://doi.org/ 10.1016/j.psychres.2020.113190

Martinez-Ferran M, de la Guía-Galipienso F, Sanchis-Gomar F, ParejaGaleano H (2020) Metabolic impacts of confinement during the COVID-19 pandemic due to modified diet and physical activity habits. Nutrients 12(6):1549. https://doi.org/10.3390/nu12061549

Maugeri G, Castrogiovanni P, Battaglia G, Pippi R, D'Agata V, Palma A et al (2020) The impact of physical activity on psychological health during Covid-19 pandemic in Italy. Heliyon 6(6):e04315. https:// doi.org/10.1016/j.heliyon.2020.e04315

Park M, Cook AR, Lim JT, Sun Y, Dickens BL (2020) A systematic review of COVID-19 epidemiology based on current evidence. J Clin Med 9(4):967. https://doi.org/10.3390/jcm9040967

Pulvirenti F, Cinetto F, Milito C, Bonanni L, Pesce AM, Leodori G et al (2020) Health-related quality of life in common variable immunodeficiency Italian patients switched to remote assistance during the COVID-19 pandemic. J Allergy Clin Immunol Pract 8(6):1894.e21899.e2. https://doi.org/10.1016/j.jaip.2020.04.003

Randolph HE, Barreiro LB (2020) Herd immunity: understanding COVID-19. Immunity 52(5):737-741. https://doi.org/10.1016/j. immuni.2020.04.012

Salari N, Hosseinian-Far A, Jalali R, Vaisi-Raygani A, Rasoulpoor S, Mohammadi M et al (2020) Prevalence of stress, anxiety, depression among the general population during the COVID-19 pandemic: a systematic review and meta-analysis. Glob Health 16(1):57. https:// doi.org/10.1186/s12992-020-00589-w

Stanton R, To QG, Khalesi S, Williams SL, Alley SJ, Thwaite TL et al (2020) Depression, anxiety and stress during COVID-19: associations with changes in physical activity, sleep, tobacco and alcohol use in Australian adults. Int J Environ Res Public Health 17(11): 4065. https://doi.org/10.3390/ijerph17114065

Tangye SG, Al-Herz W, Bousfiha A, Chatila T, Cunningham-Rundles C, Etziono A et al (2020) Human inborn errors of immunity: 2019 update on the classification from the International Union of Immunological Societies Expert Committee. J Clin Immunol 40(1):24-64. https://doi.org/10.1007/s10875-019-00737-x

Terpos E, Ntanasis-Stathopoulos I, Elalamy I, Kastritis E, Sergentanis TN, Politou $\mathrm{M}$ et al (2020) Hematological findings and complications of COVID-19. Am J Hematol 95(7):834-847. https://doi.org/ 10.1002/ajh.25829

Tian W, Jiang W, Yao J, Nicholson CJ, Li RH, Sigurslid HH et al (2020) Predictors of mortality in hospitalized COVID-19 patients: a systematic review and meta-analysis. J Med Virol 92(10):1875-1883. https://doi.org/10.1002/jmv.26050 Advance online publication

Torales J, O'Higgins M, Castaldelli-Maia JM, Ventriglio A (2020) The outbreak of COVID-19 coronavirus and its impact on global mental health. Int J Soc Psychiatry 66(4):317-320. https://doi.org/10.1177/ 0020764020915212

Vindegaard N, Benros ME (2020) COVID-19 pandemic and mental health consequences: systematic review of the current evidence. Brain Behav Immun Health 89:531-542. https://doi.org/10.1016/j. bbi.2020.05.048

Wang J, Lee YF, Liu F \& Zhou M (2020) To relax restrictions: Are communities ready to deal with repeated epidemic waves of COVID-19? Infect Cont Hosp Epidemiol 42(2):239-240. https:// doi.org/10.1017/ice.2020.228

Whitaker C, Stevelink S, Fear N (2017) The use of Facebook in recruiting participants for health research purposes: a systematic review. J Med Intern Res 19(8):e290. https://doi.org/10.2196/jmir.7071

Xu S, Li Y (2020) Beware of the second wave of COVID-19. Lancet 395(10233):1321-1322. https://doi.org/10.1016/S0140-6736(20) 30845-X

Zheng Z, Peng F, Xu B, Zhao J, Liu H, Peng J et al (2020) Risk factors of critical \& mortal COVID-19 cases: a systematic literature review and meta-analysis. J Inf Secur 81(2):e16-e25. https://doi.org/10. 1016/j.jinf.2020.04.021

Publisher's note Springer Nature remains neutral with regard to jurisdictional claims in published maps and institutional affiliations. 